\title{
Factores condicionantes de la calidad del aprendizaje en MasterFUEGO Factors that affects the quality of learning in MasterFUEGO
}

\author{
Javier Blanco Fernández ${ }^{1}$, Marta Fajó-Pascual ${ }^{2}$, Domingo Miguel Molina-Terrén ${ }^{1}$ \\ analista.incendios@gmail.com,mfajo@unizar.es,dmolina@pvcf.udl.es \\ ${ }^{1}$ Departamento de Producción Vegetal y Ciencia \\ Forestal \\ Universidad de Lleida \\ Lleida, España \\ ${ }^{2}$ Facultad de Ciencias de la Salud y del Deporte \\ Universidad de Zaragoza \\ Huesca, España
}

\begin{abstract}
Resumen- La misión de MásterFUEGO es formar técnicos con alta especialización en incendios forestales, a través de la ampliación del conocimiento científico, con el objetivo de obtener profesionales competentes en gestión de emergencias forestales. MasterFUEGO lo lidera la Universidad de Lleida (UdL) y completan Universidad de Córdoba (UCO) y Universidad de León (ULE). Con 90 créditos ECTS, es título compartido entre las tres y Oficial por el Ministerio Español de Educación. Es máster semi-presencial constituido por 7 periodos de 5 jornadas presenciales. Los factores condicionantes de la calidad del aprendizaje se han evaluado mediante una encuesta que rellenan los egresados y aquellos estudiantes que ya tienen suficiente conocimiento sobre el Máster (>40 créditos superados). De 129 alumnos potenciales de las cuatro primeras cohortes, obtuvimos un $81,4 \%$ de tasa de respuesta. El $84,7 \%$ de los encuestados se encuentran en situación laboral activa y el 68,5\% declaran que su empleo versa sobre incendios forestales. La misión queda cumplida al constatarse que los que reportan haber adquirido mayor nivel en competencias y habilidades provienen de titulaciones forestales y llevan más de 6 años de experiencia. Asimismo, "Técnicos o Mandos Superiores" y "Mandos Intermedios" suponen un 50,5\% y un 26,7\% respectivamente.
\end{abstract}

Palabras clave: Evaluación, aprendizaje, competencias, habilidades, máster, Universidad, emergencias, incendios

\begin{abstract}
MasterFUEGO's mission is to train fire professionals to the best specialization level, through sound scientific knowledge, to obtain proficient professionals in wildland fire emergency management. This program is led by University of Lleida (UdL) and both University of Cordoba (UCO) and University of León (ULE) complete the team. With 90 ECTS credits, it is joint title of the three and official by the Spanish Ministry of Education. It is a largely online program with 7 Face-to-Face training periods of 5 days. Factors conditioning the quality of learning have been evaluated by a survey completed by graduates and those students who already have sufficient knowledge about the program ( $>40$ credits completed). Of 129 potential students from the first four cohorts, we obtained an $81.4 \%$ response rate. $84.7 \%$ of the respondents are in an active labor situation and $68.5 \%$ declare that their work deals with wildland fires. The mission is fulfilled because those who report having acquired a higher level in competences and skills come from forestry degrees and have more than 6 years of experience. Likewise, "Technicians or Superior Controls" and "Intermediate Controls" account for 50.5\% and $26.7 \%$ of respondents respectively.
\end{abstract}

Keywords: Assessment, learning, competencies, skills, know-how, master, University, emergencies, fires

\section{INTRODUCCIÓN}

MásterFUEGO contribuye a la formación de personal altamente cualificado para cumplir con la demanda social de gestión de las emergencias por incendios forestales. La gestión de los programas de defensa contra incendios forestales, y en particular, las actividades vinculadas con las operaciones de extinción, están directamente relacionadas con la aplicación del sistema de manejo de emergencias (SMEIF). La universidad líder es Universidad de Lleida (UdL) y completan otras dos: Universidad de Córdoba (UCO) y Universidad de León (ULE). Es de 90 créditos ECTS. Título Oficial de Máster del Ministerio Español de Educación. Título de máster conjunto de las tres universidades. La web oficial es: http://www.masterfuegoforestal.es.

Resaltamos que es un máster semi-presencial:

A. Consta de 7 periodos de 5 días consecutivos y presenciales que engloban algunos cursos optativos (no todos hay que cursarlos). Tres periodos son en UdL, dos en UCO y otros dos en ULE. La presencialidad tiene un aprendizaje basado en problemas en seminarios y aprendizaje basado en proyectos (Prieto, Díaz \& Santiago, 2017) como los que se encargar como tareas evaluables posteriores.

B. Exige trabajos vía plataforma Moodle previo y posterior a esa presencialidad limitada en el tiempo. Y esto se ha diseñado para favorecer la matrícula de personas ya en el mercado laboral. Todas los trabajos van encaminados a evaluar las competencias a adquirir (Villadrón, 2006).

C. Más de la mitad de los alumnos lo son a tiempo parcial y lo completan en tres o más años académicos.

En cuanto a referencias de implantación del máster:

A. Hay 80 alumnos egresados en MásterFUEGO (hasta junio 2017). Y 125 con más de 60 créditos superados los 90 del máster.

B. Precedentes: Desde 2003, hemos tenido un máster análogo como título propio de la UdL, con 60 créditos ECTS. Hay 98 egresados de ese máster que se cerró para dar paso al nuevo oficial.

C. Internacionalización: Hay alumnos de otros países europeos Italia, Portugal, Alemania y Andorra. Y de América: Argentina, Venezuela, Colombia, Uruguay y 
México. Y de África: Guinea Conakry. Alumnos españoles han realizado prácticas en Chile, Argentina, Italia y Estados Unidos.

D. La evaluación de la calidad realizada por AQUANECA, en 2015, nos otorgó la máxima calificación posible como se ve en Calidad de los resultados formativos (n.d.).

E. Patrocinadores: Hay bastantes (entre ellos Vallfirest, Tecnoslylva, Diputación de Lleida, Pau Costa Fundation, Junta de Castilla la Mancha y Budenheim) como se ve en Patrocinadores de masterFUEGO (n.d.), luego parece útil para la sociedad

\section{CONTEXTO}

Con la perspectiva de cuatro cohortes ya egresadas o en vías de finalización, se resalta la necesidad de evaluar y adecuar el aprendizaje a las necesidades de la práctica profesional. Y, para ello, es fundamental conocer la opinión de quienes han cursado el máster y, en particular, aquellos que están en el mercado laboral. El objetivo es verificar si se adquieren (y cuantitativamente en qué grado) las competencias y habilidades explicitadas en la memoria verificada del título. Hay tres factores de contexto:

\section{A. Primer factor: definición de misión y visión}

MásterFUEGO tiene como misión el formar técnicos con alta especialización en incendios forestales, a través de la ampliación de conocimiento sobre los diferentes aspectos de ciencia, recursos forestales $\mathrm{y}$ emergencias por incendios forestales con el objetivo de obtener profesionales cualificados y con competencias en gestión de emergencias forestales. Esos técnicos son nuestro público objetivo.

La visión de MásterFUEGO es aunar los esfuerzos de tres universidades (y sus equipos humanos) con diferentes organizaciones (administraciones y empresas) líderes en el sector tanto en España como a nivel Internacional para proveer una formación del más alto nivel y con enfoque internacional. Unos cuantos alumnos han realizado prácticas en Chile, Argentina, Italia y Estados Unidos. Pero también muchas prácticas de gran calidad en España en dispositivos forestales o de emergencias (i.e., Cataluña, Aragón, Navarra, Valencia , Baleares, Andalucía, Castilla La Mancha, Canarias, Galicia, Asturias y Murcia), así como en tres empresas de proyectos de ingeniería y en varios centros de I+D+i. Es también la visión de MásterFUEGO que una cierta diversidad del alumnado proporcione más calidad educativa permitiendo el aprendizaje entre pares tanto en la discusión en clase y en las salidas al campo, como en la realización de trabajos en grupos multidisciplinares. Esto significa la admisión de alumnos internacionales, graduados de sectores diferentes al forestal, alumnos jóvenes graduados y también seniors en el mundo laboral. Estos últimos incluyen Jefes de Servicio, Jefes de Sección, y Directivos y Dueños de empresas de proyectos de ingeniería.

\section{B. Segundo factor: contexto social organizativo}

En la figura 1, se muestra el contexto social organizativo, la operacionalización a través de diversos procesos para lograr la visión y misión. Los actores principales son docentes, estudiantes y personal de administración y servicios. Esto condicionado por nuestra infraestructura y recursos tanto físicos como informáticos.

En el proceso de operacionalización se pasa de un concepto teórico, que manejamos en el planteamiento de la investigación y que generalmente es muy difícil de medirlo en la realidad directamente, hasta un concepto empírico, transformado por el investigador para poderlo medir en la realidad social y que consta de Concepto, Dimensión, Indicador y Categoría:

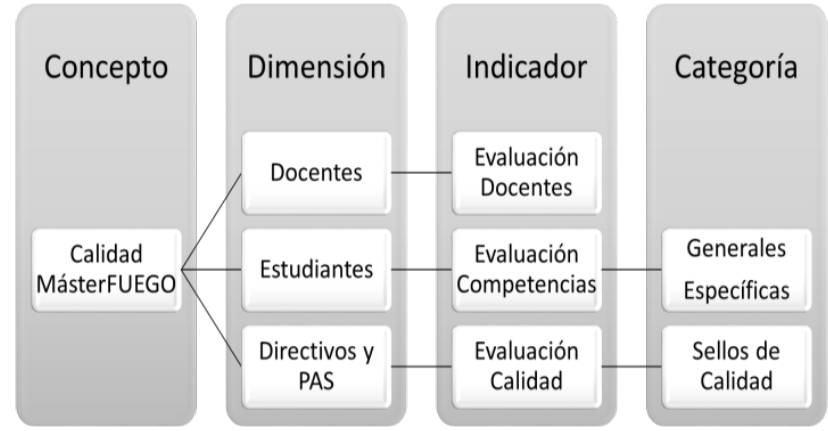

Figura 1 Proceso de operacionalización del concepto "Calidad MásterFUEGO". Elaboración propia a partir de Operacionalización: dimensiones, indicadores y variables (n.d.) de Francisco Francés García (Departamento Sociología II, Universidad de Alicante).

Investigamos si hemos sido eficaces en proporcionar las competencias tanto generales como específicas. Y lo hacemos a partir de las encuestas al egresado y alumnos con más de 40 créditos superados en los términos que se detallan en los sucesivos apartados. La contribución específica del docente en aprendizaje no ha sido considerada en este estudio. Eso es objeto habitual de las encuestas oficiales de cada asignatura.

\section{Tercer factor: medio externo}

Analicemos el medio externo al consorio MásterFUEGO (la unión de las tres universidades). Presenta tres subfactores:

- Entorno cultural: El máster busca la formación continuada de profesionales (mandos de los equipos de extinción) y la formación avanzada de profesionales recién titulados para que puedan acceder mejor al exigente mercado laboral.

- Entorno académico: Es un máster oficial al que se accede a partir de una titulación universitaria. Y no son solo universitarios del sector forestal. Esto supone retos por la procedencia dispar pero permite crecer al tener distintas disciplinas que es positivo para el aprendizaje. Este se nutre de la discusión en clase entre pares con misma (o no) formación base y cómo no de las numerosas salidas al campo.

- Entorno socioeconómico: Hay un régimen de semipresencialidad y se concentra en 7 semanas. Así es razonable para los profesionales que son el cuerpo primordial del máster.

El público objetivo de este estudio es a) egresado y alumno potencial en el futuro, b) empleador y c) el profesorado de este programa y los técnicos del servicio de calidad.

\section{DESCRIPCIÓN}

El proceso de investigación de los factores condicionantes de la calidad del aprendizaje se plantea a través de la realización de una encuesta de valoración que pueden rellenar todos los estudiantes del máster, desde los ya egresados, hasta aquellos que ya tienen un conocimiento suficiente y que en su caso se ha cuantificado en 40 ECTS. 
El presente trabajo se estructura en tres partes: "Instrumento de evaluación”, "Elaboración de la herramienta” y “Análisis”:

La primera de ellas trata sobre el tipo de instrumento de evaluación que ha sido un Trabajo de Fin de Máster que pretendía investigar sobre la calidad del aprendizaje y en qué medida se ajustaba o separaba de la memoria docente del master. La segunda parte se centra en la elaboración de la herramienta de toma de datos, la Encuesta de Evaluación, estructurada en cuatro grandes grupos:

1. Tipo de Estudiante: Se clasifica en función de la cohorte a la que pertenece el alumno, región de procedencia, créditos ECTS ya superados, edad y estudios previos. Por estudios previos entendemos a) si es de formación forestal o no y b) si el nivel previo es grado o bien título de 5 o 6 años (o master).

2. Empleo: Se estudia la situación laboral actual, tipo de actividad económica, funciones, tipo de contrato y motivación para cursar MásterFUEGO.

3. Competencias Generales y Específicas: Se mide la satisfacción con la adquisición de las competencias, el nivel necesario en el trabajo que desarrolla el egresado en ellas y la contribución del máster a su desarrollo.

4. Valoración General: Se pide una valoración general del aprendizaje, así como propuestas concretas de mejora en los contenidos de las asignaturas y en las competencias objetivo. Y si hacen falta otras asignaturas, contenidos o competencias.

La tercera parte se centra en el análisis estadístico descriptivo de los datos. El cuestionario está dirigido a los egresados y alumnos entre los años 2012 y 2016. En este punto, cabe diferenciar entre el "Material de partida" que serían todas las encuestas conseguidas del total de alumnos y egresados que ha tenido MásterFUEGO durante los diferentes años y la "Caracterización del Encuestado, Egresado y Alumno” donde se verán los rasgos más importantes de cada uno de los diferentes grupos de alumnos.

\section{A. Material de partida}

Son 129 alumnos (los ya egresados y aquellos que ya tienen superados 40 créditos) de las cuatro primeras cohortes (MF1, MF2, MF3, y MF4) distribuidos como se ve en la tabla 1.

Tabla 1 Número de alumnos totales y encuestados por cohorte

\begin{tabular}{|c|c|c|c|c|}
\hline Cohorte Cursos & Total & Encuestados & Porcentajes \\
\hline MF 1.0 & $2012-13$ & 42 & 32 & $\mathbf{7 6 , 1 9 \%}$ \\
\hline MF 2.0 & $2013-14$ & 26 & 20 & $\mathbf{7 6 , 9 2 \%}$ \\
\hline MF 3.0 & $2014-15$ & 33 & 33 & $\mathbf{1 0 0 , 0 0 \%}$ \\
\hline MF 4.0 2015-16 & 28 & 20 & $\mathbf{7 1 , 4 3 \%}$ \\
\hline TOTALES & 129 & $\mathbf{1 0 5}$ & $\mathbf{8 1 , 4 0 \%}$ \\
\hline
\end{tabular}

B. Caracterización del encuestado: egresado y alumno

Han sido 105 los alumnos que han cumplimentado la evaluación de un máximo posible de 129. Por tanto, tenemos una tasa media de respuesta del 81,4\%.

Se registró género, procedencia geográfica, edad, título de acceso al máster, situación laboral, actividad económica, funciones laborales, tipo de contrato y experiencia en el sector. Los egresados son un 45,7 \% de los encuestados. Entre los egresados encuestados tenemos 39 hombres y 9 mujeres, o $81 \%$ y $19 \%$ respectivamente. El perfil mayoritario de los hombres corresponde a edad entre 30 años y 45 años y con experiencia en el sector de más de 11 años pero no superior a 20 años. (24,5\%). El perfil mayoritario de las mujeres corresponde a una edad entre 35 y 45 años (44,4\%) y con experiencia laboral variable. Esto se resume en la tabla 2.

Los alumnos “>60 créditos superados" son un $40 \%$ de los encuestados. Tenemos 30 hombres y 12 mujeres, o $71 \%$ y $29 \%$ respectivamente. El perfil mayoritario de los hombres corresponde a edad entre 30 años y 45 años y con experiencia en el sector de más de 11 años. (26,8\%). El perfil mayoritario de las mujeres corresponde a una edad entre 25 y 34 años y experiencia inferior 2 años (58,3\%).

Los alumnos “40-60 créditos superados” son un 14,3\% de los encuestados. Tenemos 11 hombres y 4 mujeres, o $73 \%$ y $27 \%$ respectivamente. El perfil mayoritario de los hombres corresponde a edad entre 35 años y 45 años y con experiencia en el sector de más de 11 años. (63,6\%). El perfil mayoritario de las mujeres corresponde a una edad entre 30 y 34 años y con experiencia entre 7 y 10 años (50\%).

Tabla 2 Resumen de la caracterización del Egresado y del Alumno

\begin{tabular}{|c|c|c|}
\hline & Hombres (años) & Mujeres (años) \\
\hline Egresados & $\begin{array}{c}\text { Edad } 30-45 \\
\text { Experiencia } 11-20\end{array}$ & $\begin{array}{c}\text { Edad } 35-45 \\
\text { Experiencia variable }\end{array}$ \\
\hline $\begin{array}{c}\text { Alumnos }>60 \\
\text { créditos }\end{array}$ & $\begin{array}{c}\text { Edad } 30-45 \\
\text { Experiencia }>11\end{array}$ & $\begin{array}{c}\text { Edad } 25-34 \\
\text { Experiencia }<2\end{array}$ \\
\hline $\begin{array}{l}\text { Alumnos } 40 \text { - } \\
60 \text { créditos }\end{array}$ & $\begin{array}{c}\text { Edad } 35-45 \\
\text { Experiencia }>11\end{array}$ & $\begin{array}{c}\text { Edad } 30-34 \\
\text { Experiencia } 7-10\end{array}$ \\
\hline
\end{tabular}

\section{La encuesta}

Para poder mejorar la oferta de aprendizaje, se ha solicitado encarecidamente la colaboración del alumnado, con la máxima veracidad en las respuestas, asegurándoles la más absoluta confidencialidad de sus opiniones. Esta encuesta contiene 56 entradas entre preguntas y sub-preguntas y michas son tipo Likert con valores del 0 al 10)

\section{Las competencias generales y específicas}

El cuestionario mide la satisfacción con el aprendizaje analizando las competencias, el nivel necesario de ellas en el trabajo que desarrolla el encuestado y la contribución del máster a su desarrollo. Y son las siguientes.

\section{Competencias generales:}

- He perfeccionado mi disciplina (operaciones, coordinación, logística, planificación, etc.).

- He desarrollado mi capacidad de comunicación.

- Soy capaz de aplicar con rapidez los nuevos conocimientos.

- He desarrollado la capacidad de integrar conocimientos y enfrentarme a la complejidad de formular juicios a partir de la información suministrada.

- He ampliado mi capacidad de coordinar diversas actividades de forma simultánea.

- He desarrollado mi capacidad de trabajar en equipo.

- He mejorado mi capacidad de gestionar y organizar mi tiempo de estudio, adquiriendo la responsabilidad de mi propio aprendizaje.

- Movilizo mejor las capacidades de otros compañeros. 
- Utilizo mejor las diferentes herramientas informáticas (ofimática, web, e-mail, PDF). Y siguen 4 competencias más (omitidas en este texto breve).

\section{Competencias específicas:}

- He mejorado mi capacidad de predicción del comportamiento del fuego e interpretar su significado.

- He mejorado mi capacidad de análisis del comportamiento del fuego.

- He generado capacidad de resolución de problemas en ambientes de estrés durante la gestión de una emergencia por incendio forestal.

- He adquirido la capacidad de definir, organizar y dirigir los recursos humanos y materiales que intervienen en una emergencia teniendo en cuenta los distintos papeles y roles de los operativos.

- He desarrollado la capacidad de elegir y seleccionar las mejores estrategias y tácticas de extinción a partir de una información existente.

- He mejorado mi capacidad de redactar documentos de contenido técnico, informes, proyectos, o documentos de planificación, que conllevan evaluación de alternativas y cálculo de costes económicos. Y siguen 13 competencias más (omitidas en este texto breve).

Finalmente se pide una valoración de la formación recibida mediante las respuestas a un conjunto de preguntas y subpreguntas (que son en total 23).

\section{Resultados}

De un total de 129 alumnos y egresados, hemos obteniendo 105 encuestas completas. De estas, el $85,7 \%$ son ya egresados o bien han completado más de 60 créditos con lo que disponen de una notable capacidad de valorar el aprendizaje en este máster y por ello permitir un fuerte impacto.

A continuación, se exponen los resultados extraídos sobre la caracterización del encuestado, egresado y alumno:

- Tasa de respuesta: se ha obtenido una tasa de respuesta del 81,4\% de 129 alumnos, llegando en el caso del curso 20142015 al 100\% de los alumnos y no siendo inferior al 71,4\%. Luego, es una muestra muy representativa y de fuerte impacto.

- Género: cabe destacar que el 23,8\% de los encuestados son mujeres dentro de un sector mayoritariamente cubierto por hombres. Por tanto este máster sí motiva e incorpora a mujeres y esto es un valor a valorar, conservar y potenciar.

- Procedencia geográfica: El 18,1\% de los encuestados que proceden de Castilla la Mancha, seguidos de Andalucía, Cataluña y la Comunidad Valenciana con un $11,5 \%$ cada una. Es bastante uniforme. Solo es escasa la participación de Madrid y Galicia La menor de Canarias se justifica porque su insularidad exige mucha movilidad y planificación. No tenemos presencia de alumnos del País Vasco. Esto razonable ya que es la CCAA con menor incidencia por incendios forestales (MAPAMA, n.d.). Solo hay encuestados 3 de 105 de fuera de España.

- Edad: El 73.4 \% tiene más de 30 años. Así que se puede concluir que sí captamos la atención del colectivo más senior.
- Título de Acceso al Máster: si bien el sector de los incendios forestales ha sido casi exclusivo para ingenieros de la rama forestal/montes, ya observamos un cambio. El 14\% corresponde a estudiantes ajenos a "forestal/montes".

- Situación Laboral y Tipo de Contrato: el 84,7 \% de los encuestados se encuentran en situación laboral activa y, además, el 68,5 \% declaran que su trabajo está relacionado con MásterFUEGO. Esto implica un beneficio general importante para todos los alumnos, el traspaso de experiencias también entre el alumnado de diferentes procedencias.

- Un 70,5 \% de los encuestados creen que MásterFUEGO sirve para mejorar su empleo (29,5 \% que piensan que no). El $19 \%$ son empleados de la Administración pública cuya situación laboral está caracterizada por su estabilidad.

- Actividad Económica: uno de los objetivos principales era llegar a aquellos profesionales del sector ajeno a las Administraciones Públicas ya que éstas, por ley, están obligadas a favorecer la formación de los empleados públicos. Se ha conseguido ya que un $48,6 \%$ de los encuestados trabajan en empresas del sector forestal o emergencias. No todas las empresas fomentan y/o participan activa y económicamente en la formación de sus trabajadores. Estos trabajadores, en ocasiones, son los que invierten en su propia formación.

- Funciones Laborales: otro de los objetivos que MásterFUEGO se planteó en sus orígenes fue captar la atención de “Técnicos o Mandos Superiores” y "Mandos Intermedios”. Se ha logrado ya que (en las encuestas) estos son un $50,5 \%$ y un $26,7 \%$ respectivamente. Ahora bien, en el sector de los incendios forestales, existe una cierta infracontratación laboral por falta de plazas de gestión técnica en relación al número de puestos de mandos intermedios. Esto es no aprovecha completamente la capacidad del trabajador al contratarle en puestos para los que está sobre-cualificado.

Experiencia: Sobre si capta la atención de profesionales del sector que buscan el contacto y la transmisión de la experiencia de otros profesionales. El 52,4\% de los encuestados tienen una experiencia superior a 7 años en el sector y el 73,4 \% superior a 2 años.

En el estudio, se han establecido diferentes grupos de comparación por tal de contrastar opiniones sobre el máster:

- Por Finalización: Egresados vs. >60 ECTS vs. 40-60 ECTS

- Por Cohorte: MF1.0 vs. MF2.0 vs. MF3.0 vs. MF4.0

- Por Género: hombres vs. mujeres

- Por Edad: menores vs. mayores de 35 años

- Por Experiencia en el sector: menos vs. más de 6 años

- Contrato laboral: Administración vs. Empresa

En el cuestionario hay dos preguntas específicas sobre la motivación para estudiar nuestro máster. Se pedía valorar de 0 a 10 de forma independiente. Estas son: a) Ampliación de estudios y b) Mejora de expectativa laboral.

En cuanto a las competencias, los alumnos valoran bien los trabajos de las asignaturas. Por un lado los ensayos cortos (position papers) para adquirir maestría en la comunicación escrita. Y se valora (califica) más cuando lo hacen en inglés (hacia la competencia en inglés). Por otro lado, los trabajos basados en resolución de problemas (Redacción de un plan 
completo de quemas prescritas, Redacción de un plan de restauración forestal, Estudio crítico de un accidente laboral en incendios, Valoración crítica de la estrategia en un incendio forestal, etc.) todos ellos según metodologías específicas y sólidas explicadas en clase.

Se confeccionaron unos los diagramas de barras donde se puede contrastar la valoración de todos los encuestados y los valores de los cuartiles (Q1, Q2-Mediana y Q3) segmentadas por cada grupo de estudio y motivación. Observando los resultados del primer cuartil Q1, se aprecia como el 75\% de cada uno de los grupos valora con un 8 sobre 10 la ampliación de estudios como una de las razones por la que estudió MásterFUEGO, siendo los grupos de Mujeres y la cohorte de MásterFUEGO 1.0 los que peor han valorado con un 7,5 y el grupo MásterFUEGO 2.0 el que mejor ha valorado con un 8,5. A partir del cuadro de medianas Q2 se ve como todos los grupos de estudio se sitúan en el 9 sobre 10 a excepción de las cohortes que varían entre el 8,5 de MF 1.0; 9,5 de MF 2.0; 8 de MF 3.0 y 10 de MF 4.0.

En cuanto a que la mejora de la expectativa laboral sea el motivo de iniciar el estudio de MásterFUEGO, los resultados son más heterogéneos. Es el grupo de alumnos que pertenecen a las Administraciones Públicas (AP) el que peor valora con un 3 sobre 10 (Q1). Esta decisión por parte de los alumnos de este grupo se ve justificada desde el hecho de que ya tienen una de funcionario y que MásterFUEGO le proporcionará contactos y conocimiento, pero no una mejora en las expectativas laborales. Esta diferencia se mantiene en la mediana (Q2) con un 7 sobre 10 para los de AP, y en el tercer cuartil Q3 con un valor de 8,5 sobre 10 para los AP. Quien mejor valora este apartado es “mujeres” ya que en Q1 su valor corresponde a 8,5 sobre 10 . Este hecho se mantiene en la mediana de los grupos y en el cuadro sobre el tercer cuartil Q3. Nótese que el grupo “mujeres” ha sido el que peor ha valorado el nivel de consecución de competencias generales.

En las preguntas sobre el nivel de consecución de competencias generales, se aprecia que las competencias Mejorar mi capacidad de expresión escrita y oral en castellano, Mejorar mi capacidad de expresión escrita en inglés y Mejorar mi capacidad de expresión oral en inglés son las peor valoradas por todos los grupos, hecho que se mantiene cuando se amplían el número de valores (mediana y tercer cuartil).

Las competencias específicas han estado bastante bien valoradas. Cabe destacar la baja puntuación en el primer cuartil de todos los grupos sobre las competencias específicas de Resolución de problemas en ambientes de estrés durante la gestión de una emergencia por incendio forestal y Mejorar mi capacidad lectora en inglés. La primera (estrés) es cierto que exige (para llegar a mejorar su capacidad) tener cierta facilidad al trabajo en ambiente de estrés y no todos los encuestados la tienen. La "mejora de la capacidad lectora en inglés" no parece ser suficiente a pesar de los diferentes documentos en inglés que se deben leer como base previa a las clases presenciales y/o exámenes que contienen preguntas en inglés. Esto se debe a que a) algunos alumnos ya saben suficiente inglés y b) el resto han pasado por esta exigencia de leer en inglés sin servirles de revulsivo para mejorar significativamente en inglés.

En las preguntas sobre valoración general de la formación, el $79 \%$ ha contestado que volvería a estudiar MásterFUEGO, frente al 9,5\% que estudiarían otro máster diferente a MF y el 11,4\% que no estudiarían ningún máster.

Sobre la pregunta "me ha servido para mejorar mis oportunidades de trabajo (encontrar trabajo antes, mejorar contrato, puntuar más en procesos de selección, etc.)” la valoración es más floja en los que provienen de AP, Mujeres y de la cohorte MF 1.0. Lo de la cohorte MF 1.0 se debe a que el $47 \%$ de los alumnos de este grupo, corresponde a trabajadores de la Administración Pública.

En general y sin aplicar filtros por grupos, las asignaturas obligatorias valoradas como "más interesantes" son:

\section{ANASIM: análisis y simulación de fuegos forestales}

\section{ORGGES: organización y gestión de la emergencia}

3. QUEPRE: quemas prescritas. Es importante señalar aquí que esta asignatura reúne, integra y sintetiza mucho de lo que se ve en el máster. La formación en quemas prescritas y su desarrollo histórico está en Colaco \& Molina (2010).

\section{FISECO: física y ecología del fuego.}

Asimismo, de entre las asignaturas optativas "más interesantes" hay que destacar: a) TELEAPL: teledetección aplicada y b) GEOAPL: geotecnologías aplicadas.

En general y sin aplicar filtros por grupos, las asignaturas obligatorias valoradas como "menos interesantes" son: a) ECOAPL: economía aplicada en la defensa contra incendios forestales y b) CAUFAC: causalidad, factores y modelos de análisis de riesgos.

\section{CONCLUSIONES}

\section{A. Conclusiones de la encuesta}

Las competencias específicas han estado bastante bien valoradas. Y lo mismo las generales. De la investigación de factores condicionantes de la calidad del aprendizaje (de cara a la adquisición de las habilidades y competencias) se puede concluir:

La misión establecida en MásterFUEGO para formar "técnicos con alta especialización en incendios forestales..." queda satisfecha al constatarse que los encuestados que dicen haber adquirido los mayores niveles competenciales que provienen de titulaciones forestales con más de 6 años de experiencia en el sector. En la misma línea está el hecho de que "Técnicos o Mandos Superiores” y "Mandos Intermedios” supongan el 50,5 \% y 26,7 \% respectivamente.

Una parte de la visión del máster incide en que una cierta diversidad del alumnado proporciona más calidad educativa permitiendo el aprendizaje entre pares. Este hecho parece que se cumple a partir de las valoraciones generales en las encuestas, las procedencias geográficas de los encuestados y las diferentes titulaciones universitarias de acceso al máster (14\% corresponden a titulados universitarios no forestales).

Admitimos a suficientes seniors (73.4\% de los encuestados tienen más de 30 años) ya con cierto bagaje laboral.

Sí somos eficientes en reclutar mujeres al master. Y esto es un valor a conservar y potenciar.

Nos nutrimos de alumnos de toda España y de forma bastante balanceada. 
El alumnado ha hecho una apuesta por la formación adicional relacionada con su empleo. El 84,7\% de los encuestados se encuentran en situación laboral activa y, además, el 68,5 \% declaran que su trabajo está relacionado con incendios, lo que implica un beneficio para todos los alumnos, el traspaso de experiencia. Los encuestados creen que este máster sirve para mejorar en su empleo (70,5\%).

Los encuestados volverían a estudiar MásterFUEGO (79\%).

En general el encuestado conoce que tipo de competencias son necesarias para el desarrollo profesional. Pero hay grupos como los "NO EGRESADOS" y los "titulados NO FORESTALES” que en su valoración (en este preciso punto) se alejan de los valores medios generales, probablemente por una información incompleta.

Para las mismas competencias, se asigna un valor más alto (de manera consistente) de "nivel requerido en el ámbito profesional" frente al "nivel adquirido en el master". Esto implica o bien que debemos mejorar o bien que el rodaje en el puesto de trabajo es necesario para llegar a los niveles requeridos en el puesto de trabajo.

Por último en cuanto competencias, quizás deberíamos potenciar los trabajo en equipo. Este esfuerzo también redundaría en que el alumno se responsabilice de su propio aprendizaje y que sea capaz de movilizar las capacidades de otros compañeros (que son dos de las competencias que no alcanzan los valores más altos).

Las asignaturas optativas salen peor valoradas, en parte, porque hay menos alumnos que las han cursado.

Las asignaturas obligatorias FISECO y RESSUP tienen menor valoración porque el perfil mayoritario del alumno es de la extinción y no de ecología ni de restauración forestal.

\section{B. Transferencias a otros contextos}

Captar profesionales senior a cursar el máster es un éxito ya que cada cohorte tiene con ellos una serie de líderes que, aun tras acabar el máster, ejercen como tales (líderes) movilizando y motivando a los compañeros de cohorte. Y esto se traduce en, por ejemplo, a) motivación a otros a matricularse en los siguientes años y b) la alta tasa de respuesta a la encuesta de valoración, un 81,4\%. Nos hemos apoyado en esos líderes para lograr esa alta tasa y también para mantener un nivel bueno de matrículas año tras año. Creemos que admitir un porcentaje significativo de seniors (al menos un 25\%) es esencial para los programas ligados al mundo profesional.

\section{Recomendaciones}

MásterFUEGO debe hacer hincapié en que el alumno desarrolle y mejore su capacidad de comunicación y exposición aprovechando los periodos de clase presencial o fomentando el empleo de las TIC; asimismo, también debe facilitar que el alumno desarrolle la capacidad de coordinar diversas actividades de forma simultánea a través la potencialización de los múltiples recursos que hay a disposición en nuestra plataforma Moodle. Para mejorar las capacidades de expresión oral, proponemos la realización de trabajos a partir de exposiciones (tanto en español como en inglés) aprovechando los días presenciales.

La asignatura de TELEME hay que reenfocarla a mayor aplicación en el sector de emergencias por incendio forestal.

La asignatura de GESINF hay que reenfocarla a un campo más próximo al Oficial de Información.

La asignatura de ORGGES debería añadir supuestos prácticos de gestión de emergencias, así como la visita a diferentes centros de Coordinación Operativa (CECOP), este hecho favorecería también el conocimiento de la gestión de una emergencia desde el interior de "las cuatro paredes".

Abriremos una optativa nueva de Meteorología que abarque conceptos básicos y avanzados que confieran una capacidad predictiva de potencial del fuego a corto y medio plazo.

\section{AgRADECIMIENTOS}

Nuestro agradecimiento a los egresados y alumnos que han puesto su interés y su tiempo en responder con veracidad a la encuesta, para que esto redunde en un máster cada vez de mejor calidad en cuanto al cumplimiento de su misión.

\section{REFERENCIAS}

Calidad de los resultados formativos (n.d.) In Master Fuego Forestal http://www.masterfuegoforestal.es/sobre-elmaster/sistema-de-calidad/

Colaco C. \& Molina DM. (2010). Learning and Training on the Use of Prescribed Burning Techniques. In Towards Integrated Fire Management. Outcomes of the European Project Fire Paradox. EFI Research Report 23, p.161173. EU-contract FP6-018505. Recuperado de http://www.efi.int/files/attachments/publications/efi_rr23 .pdf

MAPAMA (n.d.) In Estadísticas de Incendios Forestales. Recuperado de http://www.mapama.gob.es/es/desarrollorural/estadisticas/Incendios_default.aspx\#para0

Patrocinadores de masterFUEGO (n.d.) In Master Fuego Forestal http://www.masterfuegoforestal.es/sobre-elmaster/patrocinadores/

Prieto, A., Díaz D. \& Santiago R. (2014). Metodologías Inductivas: El desafío de enseñar mediante el cuestionamiento y los retos (Innovación educativa). Barcelona:Digital-text. Versión Kindle.

Operacionalización: dimensiones, indicadores y variables (n.d.). In Objetivos de aprendizaje: Tema 3 - la encuesta. Recuperado de https://personal.ua.es/es/franciscofrances/materiales/tema3/operacionalizacin_dimensiones _indicadores_y_variables.html

Villadrón Gallego, M. L. (2006). Evaluación del aprendizaje para promover el desarrollo de competencias. Educatio Siglo XXI, 24, 57-76. 